\title{
The effect of supplemental oxygen on perioperative brain natriuretic peptide concentration in cardiac risk patients - a protocol for a prosprective randomized clinical trial
}

Christian Reiterer ${ }^{1}$, Barbara Kabon ${ }^{1 *}$ (D), Markus Falkner von Sonnenburg ${ }^{1}$, Patrick Starlinger ${ }^{2}$, Alexander Taschner ${ }^{1}$, Oliver Zotti ${ }^{1}$, Julius Goshin ${ }^{1}$, Gregor Drlicek ${ }^{3}$ and Edith Fleischmann ${ }^{1}$

\begin{abstract}
Background: Elevated postoperative N-terminal pro-B-type natriuretic peptide (NT-proBNP) concentrations are predictive for cardiac adverse events in noncardiac surgery. Studies indicate that supplemental oxygen decreases sympathetic nerve activity and might, therefore, improve cardiovascular function. Thus, we will test the effect of perioperative supplemental oxygen administration on NT-proBNP release after surgery.

Methods/design: We will conduct a single-center, double-blinded, randomized trial at the Medical University of Vienna, including 260 patients with increased cardiac risk factors undergoing moderate- to high-risk noncardiac surgery. Patients will be randomly assigned to receive $80 \%$ versus 30\% oxygen during surgery and for $2 \mathrm{~h}$ postoperatively. The primary outcome will be the difference in maximum NT-proBNP release after surgery. As secondary outcomes we will assess the effect of supplemental oxygen on postoperative maximum troponin $T$ concentration, oxidation-reduction potential, von Willebrand factor concentration and perioperative fluid requirements. We will perform outcome measurements $2 \mathrm{~h}$ after surgery, on postoperative day 1 and on postoperative day 3. The NT-proBNP concentration and the oxidation-reduction potential will also be measured within $72 \mathrm{~h}$ before discharge.
\end{abstract}

Discussion: Our trial should determine whether perioperative supplemental oxygen administration will reduce the postoperative release of NT-proBNP in patients with preoperative increased cardiovascular risk factors undergoing noncardiac surgery.

Trial registration: ClinicalTrials.gov, ID: NCT03366857. Registered on 8th December 2017.

* Correspondence: barbara.kabon@meduniwien.ac.at

1 Department of Anaesthesia, General Intensive Care Medicine and Pain Medicine, Medical University of Vienna, Spitalgasse 23, 1090 Vienna, Austria

Full list of author information is available at the end of the article

C The Author(s). 2020 Open Access This article is licensed under a Creative Commons Attribution 4.0 International License, which permits use, sharing, adaptation, distribution and reproduction in any medium or format, as long as you give appropriate credit to the original author(s) and the source, provide a link to the Creative Commons licence, and indicate if changes were made. The images or other third party material in this article are included in the article's Creative Commons licence, unless indicated otherwise in a credit line to the material. If material is not included in the article's Creative Commons licence and your intended use is not permitted by statutory regulation or exceeds the permitted use, you will need to obtain permission directly from the copyright holder. To view a copy of this licence, visit http://creativecommons.org/licenses/by/4.0/ The Creative Commons Public Domain Dedication waiver (http://creativecommons.org/publicdomain/zero/1.0/) applies to the data made available in this article, unless otherwise stated in a credit line to the data. 


\section{Background}

Major cardiovascular complications occur in about 3\% of all patients undergoing noncardiac surgery $[1,2]$. In patients over the age of 45 years the risk of developing postoperative complications is even higher [2].

As supplemental oxygen has no effect on the incidence of surgical site infections, it is reasonable to question the effect of hyperoxia on other organ systems [3-6]. The systemic effect of perioperative supplemental oxygen, especially on the cardiovascular system, still remains unknown. A post-hoc analysis of the PROXI trial indicated an increased long-term risk of myocardial infarction or other heart diseases in patients receiving supplemental oxygen [7]. Despite the current controversy regarding toxic effects of supplemental oxygen [8], there are also studies indicating the beneficial effects of higher oxygen concentrations specifically on the cardiovascular system. A decrease in heart rate and myocardial oxygen consumption as well as an increase in myocardial oxygen supply was shown previously [9-11].

For example, nocturnal oxygen therapy significantly reduced the brain natriuretic peptide (BNP) concentration and prevented the progression of congestive heart failure in patients with central sleep apnea [12].

$N$-terminal pro-B-type natriuretic peptide (NTproBNP) is a hormone, which is released during increased myocardial stress [13]. Measurement of NTproBNP is recommended to enhance perioperative cardiac risk estimation in patients aged over 65 years of age or older, and also those aged 45-64 years of age with significant cardiovascular disease undergoing noncardiac surgery [14].

The preoperative assessment of NT-proBNP is recommended for patients at high risk of cardiovascular events undergoing noncardiac surgery [15]. Postoperatively elevated NT-proBNP concentrations are associated with increased postoperative cardiac mortality and cardiac failure within 30 days after noncardiac surgery [16].

No data are available regarding the effect of supplemental oxygen on postoperative NT-proBNP concentrations. Thus, we will test our hypothesis that perioperative supplemental oxygen (80\% versus $30 \%$ ) decreases the postoperative maximum NT-proBNP concentration assessed within $2 \mathrm{~h}$, on postoperative day (POD) 1 and POD 3 in patients with increased cardiovascular risk factors undergoing moderate- to high-risk noncardiac surgery. Furthermore, we will evaluate differences of NT-proBNP concentration within $72 \mathrm{~h}$ before discharge between both study groups. Our secondary outcomes will test the effect of supplemental oxygen on postoperative troponin $\mathrm{T}$ $(\mathrm{TnT})$ concentration, oxidation-reduction potential and von Willebrand factor (vWF) concentration and perioperative fluid requirements.

\section{Methods/design: data collection, management and analysis \\ Objectives and design}

We will conduct a single-center, double-blinded, two-arm trial at the Medical University of Vienna. In total, we will randomly assign 260 patients to receive $80 \%$ versus $30 \%$ inspired oxygen concentration for surgery and for $2 \mathrm{~h}$ postoperatively. We will follow the Standard Protocol Items: Recommendations for Interventional Trials (SPIRIT) recommendations for interventional trials (Additional file 1). The study was approved by the Ethics Committee of the Medical University of Vienna on 13 November 2017. This trial was registered at ClinicalTrials. gov on 8 December 2017 (NCT03388957) and at the European Clinical Trial Database (2017-003714-68).

This trial will test the primary hypothesis that supplemental oxygen significantly decreases the maximal postoperative NT-proBNP concentration (assessed within 2 h after surgery, on POD 1 and POD 3) in patients with increased cardiovascular risk factors undergoing moderate- to high-risk abdominal surgery.

Our secondary outcomes are differences in maximum TnT concentration, static oxidation-reduction potential (sORP) and oxidation-reduction potential capacity (cORP), as well as vWF values and the perioperative fluid requirements to reach hemodynamic stability (Table 1).

\section{Study population}

We will screen for patients of at least 45 years of age planned for elective moderate- to high-risk abdominal surgery under general anesthesia with an expected duration of surgery of at least $2 \mathrm{~h}$. Patients are eligible if they meet one or more the following criteria: (1) history of coronary artery disease; (2) history of peripheral arterial disease; (3) history of stroke or (4) any three of these six criteria: (a) age over 70 years, (b) undergoing major surgery, (c) history of congestive heart failure, (d) history of transient ischemic attack, (e) diabetes and currently taking an orally administered hypoglycemic agent or insulin and (f) history of hypertension. Patients will be not eligible if they meet one of the following criteria: (1) symptoms of infection or sepsis; (2) preoperative inotropic therapy; (3) patients under intensive care unit (ICU) treatment; (4) oxygen-dependent patients and (5) history of severe heart failure and/or an ejection fraction $<30 \%$. (Fig. 1).

\section{Protocol}

We will perform standardized monitoring including electrocardiogram (ECG), blood pressure and oxygen saturation $\left(\mathrm{SpO}_{2}\right)$ in all patients after arriving in the operating theater. Shortly before induction of anesthesia, patients will be randomly assigned to one of the two 
Table 1 Outcomes assessment

\begin{tabular}{lll}
\hline & Outcomes & Measurements \\
\hline Primary & Myocardial function & maxNT-proBNP \\
Secondary & MINS & maxTnT \\
& Surgical stress & Copeptin \\
& Redox stress & SORP, cORP \\
& Hemodynamic & \\
Exploratory & Cardiac failure & $50 \%$ increase in NT-proBNP \\
& Myocardial infarction & ECG change and elevated heart-specific enzymes \\
& Heart failure requiring intervention & $50 \%$ NT-proBNP increase and medical treatment \\
& New onset of cardiac arrhythmias & ECG changes requiring medical treatment and/or electric cardioversion \\
\hline
\end{tabular}

CORP oxidation-reduction capacity, ECG electrocardiogram, ICU intensive care unit, maxNT-proBNP maximal $N$-terminal pro-B-type natriuretic peptide, maxTnT maximal troponin T, MINS Myocardial Injury after Noncardiac Surgery, sORP static oxidation-reduction potential

oxygen concentrations. We will administer $100 \%$ oxygen in all patients for induction of anesthesia according to clinical standards. We will induce anesthesia using 1$3 \mu \mathrm{g} \mathrm{kg}^{-1}$ body weight (BW) fentanyl, $2 \mathrm{mg} \mathrm{kg}^{-1}$ (BW) propofol and $0.6 \mathrm{mg} \mathrm{kg}^{-1}$ rocuronium. In addition to standard monitoring, an arterial line will be inserted for direct blood pressure monitoring. A central venous line will be inserted at the discretion of the attending anesthesiologist.

Anesthesia will be maintained with sevoflurane (up to 1.5 minimum alveolar concentration (MAC)) in an oxygen gas carrier according to processed electroencephalogram (EEG)-guided anesthesia. Muscle relaxation will be given when necessary to maintain one to two mechanical twitches in response to supra-maximal stimulation (Train-of-Four stimulation, target $<75 \%$ ). Temperature will be held at a core temperature over $36^{\circ} \mathrm{C}$ using forced-air warming.

We will use esophageal-Doppler-guided (Cardiac Q, Deltex Medical, Chichester, UK) fluid management according to a previously published algorithm $[17,18]$. All patients will receive a $2 \mathrm{~mL} / \mathrm{kg}$ BW baseline infusion of balanced crystalloids. A bolus of $250 \mathrm{~mL}$ balanced crystalloids will be administered when the stroke volume decreases by more than $20 \%$ as compared to baseline values. In the case of acute bleeding or a systemic inflammatory response during surgery, volume will be administered according to fluid requirements to maintain hemodynamic stability. Blood and blood products will be administered per clinical judgment.

There will be no restrictions or prohibitions of medical care and therapeutics during hospital stay in case of enrollment.

\section{Intervention}

After endotracheal intubation we will set the inspired oxygen fraction according to randomization. Patients randomly assigned to the $80 \%$ oxygen group will receive an inspired oxygen fraction of 0.8 after intubation; those randomly assigned to the $30 \%$ oxygen will receive an inspired oxygen fraction of 0.3 in air. Patients will be blinded to group allocation.

We will use $80 \%$ versus $30 \%$ oxygen concentration to receive the maximum difference between oxygen concentrations without the risk of causing damage, e.g., increased formation of atelectasis [19]. Moreover, almost all studies investigating the effect of supplemental oxygen on, for example, postoperative wound infection used $80 \%$ versus $30 \%$ inspired oxygen concentration during surgery $[5,6,20]$.

Maintaining a minimum $\mathrm{SpO}_{2}$ of at least $93 \%$ will be attempted. In the case of $\mathrm{SpO}_{2}<93 \%$ the following interventions to improve oxygenation will be performed: (1) positive end-expiratory pressure (PEEP) will be increased incrementally by $2 \mathrm{cmH}_{2} \mathrm{O}$ up to maximally $12 \mathrm{cmH}_{2} \mathrm{O}$; (2) recruitment maneuver: the inspiratory pressure will be set at $55 \mathrm{cmH}_{2} \mathrm{O}$. The PEEP will be set at $12 \mathrm{cmH}_{2} \mathrm{O}$ and respiratory rate to six breaths/min or higher. Inspiratory pressure will be set to receive a tidal volume of $6 \mathrm{~mL} / \mathrm{kg}$ ideal BW. The inspiratory ratio will be set (I:E) to $1: 1$. Inspiratory pressure will be increased until a plateau pressure reaches $40-50 \mathrm{cmH}_{2} \mathrm{O}$. After allowing three breaths while maintaining a plateau pressure of $40-50 \mathrm{cmH}_{2} \mathrm{O}$, the respiratory rate and inspiratory pressure will be set back to pre-recruitment parameters and (3) if both interventions are not sufficient to increase $\mathrm{SpO}_{2}$, then the inspiratory oxygen fraction will be elevated by 0.1 until $93 \% \mathrm{SpO}_{2}$ is reached.

\section{Measurements}

\section{Baseline information}

We will record demographic and morphometric data including age, sex, height, weight, American Society of Anesthesiologists (ASA) physical status and medical history 


\section{Inclusion Criteria}

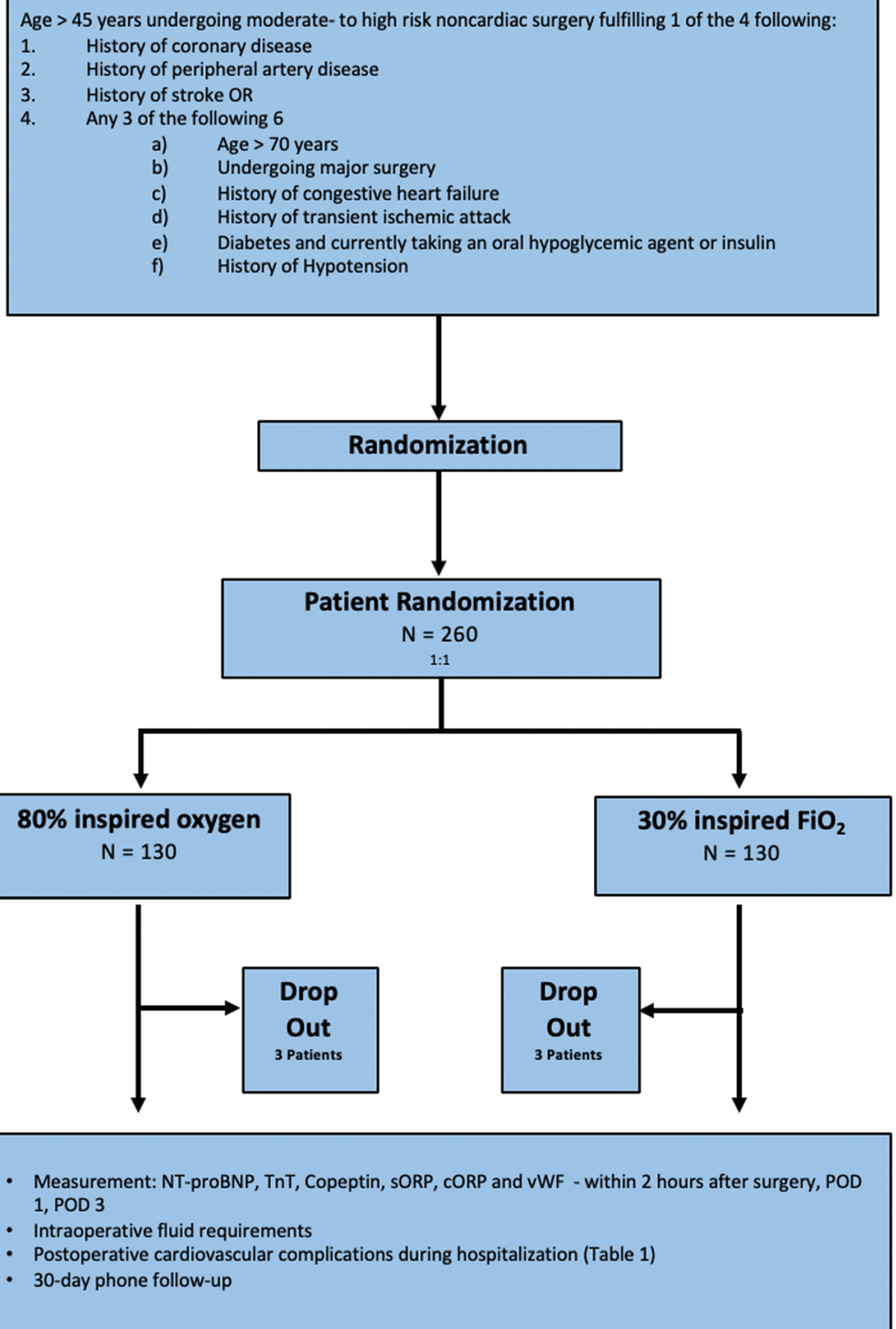

Fig. 1 Study flow chart. BNP brain natriuretic peptide, $T n T$ troponin T, sORP static oxidation-reduction potential, cORP oxidation-reduction potential capacity, POD postoperative day

(cardiovascular, pulmonary, neurological, presence of diabetes type 1 and type 2, insulin use and alcohol and tobacco use). We will record the home medication (beta-blockers, ACE inhibitors/angiotensin-1 blockers, diuretics, statins, thienopyridines, anticoagulants, insulin or orally administered antidiabetic drugs, alpha- 2 agonists/antagonists). We will perform blood samples to measure baseline values in all patients including NTproBNP, TnT (high-sensitivity, fifth-generation $\mathrm{TnT}$ ), hemoglobin, inflammatory parameters, thrombocytes, coagulation parameters, electrolytes, creatinine, albumin, liver tests and copeptin. Blood samples for NT-proBNP and oxidation-reduction will be drawn five times (preoperatively, within $2 \mathrm{~h}$ after surgery, on the first and third PODs and within 3 days of discharge). Blood samples for TnT and vWF will be drawn four times (preoperatively, within $2 \mathrm{~h}$ after surgery, on the first and third PODs).

All laboratory value will be analyzed at the Department of Laboratory Medicine at the Medical University of Vienna.

\section{Perioperative data}

Intraoperative data, such as end-tidal sevoflurane concentration, anesthetic agents used, medication, fluid balance, amount and start of vasopressor administration, 
temperature, hemodynamic and respiratory parameters (cardiac output, stroke volume and corrected flow time (FTc), $\mathrm{FiO}_{2}$ and end-tidal $\mathrm{CO}_{2}$ will be extracted from the electronic anesthesia information management system. Blood-gas analysis (oxygen partial pressure $\left(\mathrm{pO}_{2}\right)$, $\mathrm{CO}_{2}$, hemoglobin, $\mathrm{pH}$, base excess), will be performed hourly during surgery and also recorded.

\section{Follow-up}

Patients will be followed up for the primary and secondary outcome within $2 \mathrm{~h}$ after surgery, on POD 1, POD 3 and within 3 days before discharge. Laboratory measurements for outcome assessments are shown in Table 1. We will further perform a telephone follow-up on the 30th day after surgery to evaluate cardiac events including acute heart failure, myocardial infarction and new onset of atrial fibrillation (Table 2). All follow-up measurements will be performed by an investigator who will be unaware of the randomized allocation. Acute heart failure will be defined as cardiac failure needing medical intervention including therapy with inotropes and extracorporeal membrane oxygenation. Myocardial infarction will be diagnosed according the fourth definition of myocardial infarction [21]. Atrial fibrillation will be diagnosed according to the management guidelines for atrial fibrillation [22].

\section{Data analysis}

Randomized groups will be compared for balance in patient characteristics, demographic data, type of surgery, duration of surgery and anesthesia and preoperative baseline laboratory values (including: NT-proBNP, TnT, hemoglobin, hematocrit, leukocytes, thrombocytes, prothrombin time, activated prothrombin time, sodium, potassium, calcium, magnesium, creatinine, albumin, transaminases, C-reactive protein). The primary analysis will be intention to treat so that all randomized patients will be included in all analyses.

The primary outcome, the effect of $80 \%$ versus $30 \%$ oxygen on postoperative maxNT-proBNP will be compared using a two-sample, two-tailed Mann-Whitney $U$ test. If a patient dies before NT-proBNP on POD 3 is measured, the patient's maxNT-proBNP will be set to the maximum maxNT-proBNP values over all patients. Multiple imputation will be used for missing postoperative measurements.

Our secondary outcomes including Myocardial Injury after Noncardiac Surgery (MINS) (defined as absolute change of high-sensitive TnT of $5 \mathrm{ng} / \mathrm{L}$ between 20 to $<65 \mathrm{ng} / \mathrm{L}$ ) [23], surgical stress, redox stress and perioperative hemodynamic (fluid and vasopressor requirements) will be analyzed using a two-sample, two-tailed Mann-Whitney $U$ test. The exploratory outcomes including cardiac failure, myocardial infarction, acute heart failure requiring intervention, new onset of cardiac arrhythmias and unplanned ICU admission will be analyzed using Kaplan-Meier curves assessed with a log-rank test (Table 1).

Table 2 Schedule of study events

\begin{tabular}{|c|c|c|c|c|c|c|c|c|}
\hline \multirow[t]{3}{*}{ Timepoints } & \multirow{3}{*}{$\begin{array}{l}\text { Enrollment } \\
\text { Preoperative } \\
\text { visit }\end{array}$} & \multicolumn{7}{|c|}{ Study period } \\
\hline & & \multicolumn{3}{|l|}{ Intervention } & \multicolumn{3}{|c|}{ Postoperative period } & \multirow{2}{*}{$\begin{array}{l}\text { Close out } \\
\text { POD telephone } \\
\text { follow-up }\end{array}$} \\
\hline & & $\begin{array}{l}\text { Before } \\
\text { anesthesia }\end{array}$ & $\begin{array}{l}\text { During } \\
\text { surgery }\end{array}$ & $\begin{array}{l}\text { End of surgery for } 2 \mathrm{~h} \text { after } \\
\text { surgery }\end{array}$ & $\begin{array}{l}\mathrm{POD} \\
1\end{array}$ & $\begin{array}{l}\text { POD } \\
3\end{array}$ & $\begin{array}{l}\text { Hospital } \\
\text { discharge }\end{array}$ & \\
\hline \multicolumn{9}{|l|}{ Enrollment } \\
\hline Eligibility screening & $x$ & & & & & & & \\
\hline Informed consent & $x$ & & & & & & & \\
\hline Patient history & $x$ & & & & & & & \\
\hline Allocation & & $x$ & & & & & & \\
\hline \multicolumn{9}{|l|}{ Intervention } \\
\hline $80 \%$ inspired oxygen & & & $x$ & & & & & \\
\hline $30 \%$ inspired oxygen & & & $x$ & & & & & \\
\hline $\begin{array}{l}\text { Anesthesia/surgery } \\
\text { variables }\end{array}$ & & & $x$ & & & & & \\
\hline $80 \%$ via face mask & & & & $x$ & & & & \\
\hline $30 \%$ via face mask & & & & $x$ & & & & \\
\hline Adverse events & & & $x$ & $x$ & & & & \\
\hline \multicolumn{9}{|l|}{ Assessments } \\
\hline Outcomes (see Table 1) & & & & & $x$ & $x$ & $x$ & \\
\hline Adverse events & & & & & $x$ & $x$ & $x$ & $x$ \\
\hline
\end{tabular}


If hospital discharge occurs before the third POD, the maximum value of the available data will be used for outcome measure.

\section{Sample size consideration}

A previous study showed a nearly $40 \%$ reduction of BNP levels in patients with congestive heart failure receiving nocturnal oxygen therapy [12]. Based on our own data of a previous trial (unpublished data) we observed a fourfold increase of BNP (preoperative $129.05 \mathrm{ng} / \mathrm{L} \pm$ 160.13 to up to $482.49 \mathrm{ng} / \mathrm{L} \pm 538.78$ ) in the postoperative period in relatively healthy patients undergoing moderate- to high-risk open abdominal surgery. We calculated a needed sample size of 254 patients (127 patients per group) to show a $40 \%$ reduction of NTproBNP in patients receiving $80 \%$ versus $30 \%$ oxygen with a power of $80 \%$ at a 0.05 significance level. We will thus include 260 patients (130 patients per group) to compensate for potential drop outs.

\section{Assignment to intervention}

Given an average estimated recruitment rate of $10 \mathrm{pa}-$ tients per month, we anticipate a recruitment period of 25 months. A trained study coordinator will evaluate eligibility and obtain informed consent. All patients will be recruited using the operation schedule the day before surgery. After meeting the eligibility criteria, the inclusion criteria will be checked. Two copies of the signed consent form will be made. One copy remains in the patient chart, and the second one will be given to the patient. On the consent form, participants will be asked if they agree to the use of their data should they choose to withdraw from the trial. Participants will also be asked for permission for the research team to share relevant data with people from the Universities taking part in the research or from regulatory authorities, where relevant. This trial does not involve collecting biological specimens for storage.

On the day of surgery the coordinator will enroll the participants via accessing the web-based system. We will use permutated block randomization using a web-based randomization program (Randomizer, Medical University of Graz, Graz, Austria). Each block has a size of 6 numbers, randomly ordered to the two treatment assignments, of which all investigators were unaware. The web-based randomization program was set up by an investigator not involved in data acquisition. Intraoperative investigators and clinicians will be not blinded to the allocated treatment group. However, an observer strictly blinded to the group assignment will evaluate complications during hospitalization. Subsequently, a blinded investigator will call all patients 30 days after surgery and to evaluate outcomes.

\section{Data collection, management and analysis}

Data will be recorded in electronic case report forms (eCRFs) and reviewed by the clinical research associate (CRA) during monitoring visits. The CRA will verify recorded data in the electronic data

capture (EDC) system with the stored source documents. All corrections or changes made to any study data will be appropriately tracked in an audit trial in the EDC system An eCRF will be considered completed when all missing, incorrect, and/or inconsistent data have been accounted for.

For data management we will use Clincase software (Version 2.6.0.34, Quadratek, Berlin, Germany).

All records of subjects, source documents, monitoring visit logs and CRFs will be kept locked in the appropriate study files in our site.

\section{Monitoring}

The designated monitor will contact and visit the investigator on a regularly basis and will be allowed to have access to all source documents, which are needed to verify the entries in the CRF and eCRF, respectively, and other protocol-related documents. It will be the responsibility of the monitor to inspect the CRFs and eCRFs, respectively, at regular intervals according to the monitoring plan throughout the study, to verify the adherence to the proto$\mathrm{col}$ and the completeness, consistency and accuracy of the data being recorded. The monitoring will be performed by Karl Schebesta, MD, Department of Anaesthesia, Intensive Care Medicine and Pain Medicine.

\section{Access to data}

Any data required to support the protocol can be supplied on request.

\section{Ethics and dissemination Data Safety Monitoring Board}

The Data Safety Monitoring Board (DSMB) will be formed by Oliver Kimberger, MD, Department of Anaesthesia, Intensive Care Medicine and Pain Medicine, Medical University of Vienna, and Anton Stift, MD, Department of Surgery, Medical University of Vienna. The DSMB will evaluate serious adverse events (SAEs) from the trial after 100 enrolled patients. This committee, along with the Local Ethics Committee will have the exclusive authority to stop the study either because the hypothesis has been confirmed or denied, or because the adverse events are overwhelming. Any morbidity potentially related to the study protocol will be reported to the Ethics Committee.

When the data of 100 patients become available the DSMB will evaluate possible overwhelming harmful effects between both study groups and discuss whether to 
stop the trial early to prevent harm. The DSMB will monitor for an adverse impact of oxygen on SAEs.

The Trial Steering Group and the independent Data Monitoring and Ethics Committee will meet to review conduct throughout the trial period.

\section{Adjudication of the trial outcomes}

The outcome adjudicator will be blinded to treatment allocation and will adjudicate the following outcomes: death, myocardial infarction, acute heart failure, cardiac arrhythmias and unplanned ICU admission. We will use the decisions of the outcome adjudicator for all statistical analysis of these events. Barbara Kabon, MD (Department of Anaesthesia, Intensive Care Medicine and Pain Medicine) will chair the Adjudication Committee.

\section{Other management at the discretion of the attending physician}

The implementation of $80 \%$ versus $30 \%$ inspired oxygen concentration will not require alteration to usual care pathways (including use of any medication) and these will continue for both trial arms. Furthermore, there will be no anticipated harm and compensation for trial participation. There will be no restriction in the postoperative care regarding medications and therapies. All patients will be treated after completion of the study according to standard clinical care.

All aspects of the patient's management are at the discretion of the attending physician. This includes all decisions of the intraoperatively used oxygen concentration.

Blinded research personnel will follow patients throughout their time in hospital evaluating the patients and reviewing their medical records ensuring that trial orders are followed and noting any outcomes. If patients indicate that they have experienced any of the outcome measures, the study personnel will obtain the appropriate documentation.

\section{Reporting of serious adverse events (SAE)}

Serious adverse events are those which are fatal, lifethreatening or fulfill the definition of being clinically important. Efficacy or safety outcomes will not be considered as SAEs, except if, because of the course of severity or any other feature of such events, the investigator, according to their best medical judgment, considers these events as exceptional in this medical condition. All events considered as part of the primary, secondary, or safety events should be reported on the appropriate page(s) in the CRF but not as an SAE, unless considered exceptional in this medical condition. In this trial, the following events (pulmonary failure, cardiac failure, myocardial infarction, acute heart failure, new onset of cardiac arrhythmias, unplanned ICU admission) are considered related to the underlying cardiovascular disease and are not considered as a SAE. These events will not be considered unexpected unless their course, severity or other specific features are such that the investigator, according to their best medical judgment, considers these events as exceptional in the context of the patient. Only unexpected and not previously described SAEs that are believed with a reasonable level of certainty to be associated with the trial medication need to be reported immediately (i.e., within $24 \mathrm{~h}$ of knowledge of the event) to the sponsor of the trial, the Medical University of Vienna. For such events, research personal will complete the SAE CRF immediately. Regulatory authorities will be informed in a timely manner according to the applicable regulations.

\section{Discussion}

This study is designed to evaluate the effect of perioperative supplemental oxygen administration on postoperative NT-proBNP concentrations in patients with increased cardiovascular risk factors undergoing moderate- to high-risk noncardiac surgery. The physiological background of supplemental oxygen is to improve myocardial tissue oxygenation and simultaneously reduce myocardial oxygen consumption mediated by a reduction of the heart rate and cardiac output [11]. A recently published subanalysis of a large clinical trial showed a $30 \%$ reduction in the composite of myocardial injury, in hospital cardiac arrest and in mortality in patients undergoing colorectal surgery assigned to the $80 \%$ oxygen group [24]. Furthermore, elevated BNP and/or TnT concentrations in the immediate postoperative period are predictive of long-term outcome [16]. Interestingly, a subanalysis of a large outcome trial [25] showed that NT-proBNP and TnT are significantly increased in relatively healthy patients (unpublished data). It seems likely that the stress exposure due to surgery and anesthesia might restrain myocardial contractility, which will be reflected by the elevated NT-proBNP values. Despite the controversy regarding the harmful effects of supplemental oxygen during surgery there is evidence that oxygen might beneficially affect the cardiovascular system. In patients with central sleep apnea, for example, oxygen therapy attenuated the progression of chronic heart failure, which was assessed using BNP measurement [12]. Data of postoperative NT-proBNP concentrations, especially in patients with increased cardiovascular risk factors, are still lacking and are, therefore, of major interest. Thus, we are testing a possible beneficial effect of supplemental oxygen on perioperative myocardial function, which will be obtained by consecutive postoperative NTproBNP measurements.

Since the effect of hyperoxia on oxidative stress is based mostly on weak studies with small sample sizes we also decided to evaluate the effect of supplemental 
oxygen on oxidation-reduction potential [26-28]. Measurements of sORP and cORP include all known and unknown oxidants; hence, the determination of sORP and cORP should provide a comprehensive overview of the actual system of oxidative stress.

We wish further to evaluate the immune-modulating effect of supplemental oxygen on endothelial-derived factors [29, 30]. Increased inflammation mediated by hyperoxia might result in elevated vWF-antigen concentrations reflecting endothelial damage.

Previous studies in healthy volunteers indicated a dose-dependent peripheral arterial vasoconstriction, consequently increasing systemic vascular resistance [31]. Thus, we hypothesize that intraoperative supplemental oxygen administration leads to vasoconstriction, which might reduce the required amount of fluid to maintain hemodynamic stability.

\section{Summary}

We plan a randomized clinical trial in patients with increased cardiovascular risk factors undergoing moderate- to high-risk noncardiac surgery to compare the effects of intraoperative $80 \%$ inspired oxygen versus $30 \%$ inspired oxygen on postoperative maximum NT-proBNP concentrations. Since elevated postoperative NTproBNP is a strong predictor for postoperative cardiac complications, patients may benefit if the postoperative NT-proBNP release can be reduced by the simple administration of supplemental oxygen.

\section{Trial status}

Actual protocol version 1.4; 19 July 2017. Recruitment started 1 December 2017 and was completed in December 2019 .

\section{Supplementary information}

Supplementary information accompanies this paper at https://doi.org/10. 1186/s13063-020-04336-9.

Additional file 1. Standard Protocol Items: Recommendations for Interventional Trials (SPIRIT) 2013 Checklist: recommended items to address in a clinical trial protocol and related documents.

\begin{abstract}
Abbreviations
BNP: Brain natriuretic peptide; BW: Body weight; cORP: Oxidation-reduction potential capacity; DSMB: Data Safety Monitoring Board; FTc: Corrected flow time; MAC: Minimum alveolar concentration; MINS: Myocardial Injury after Noncardiac Surgery; NT-proBNP : N-terminal pro-B-type natriuretic peptide; PEEP: Positive end-expiratory pressure; $\mathrm{pO}_{2}$ : Oxygen partial pressure; POD: Postoperative day; SAE: Serious adverse event; sORP: Static oxidationreduction potential; TnT: Troponin T; vWF: von Willebrand factor
\end{abstract}

\section{Sponsor}

Medical University of Vienna

Department of Anaesthesia, Intensive Care Medicine and Pain Medicine

Spitalgasse 23, 1090 Vienna, Austria

Contact: Prof. Klaus Markstaller
The sponsor played no part in study design; collection, management, analysis and interpretation of data; writing of the report; and the decision to submit the report for publication.

\section{Dissemination}

The study is planned to be submitted for publication in a peer-review journal.

\section{Authors' contributions}

CR, BK and EF: concept and study design. CR, BK, EF, PS, AT, JG and OZ: first draft of the manuscript and data collection. JG, GD and AT: data management. CR, BK, MS, PS, AT, OZ, JG, GD, and EF: editing and critical review of the manuscript. All authors read and approved the final manuscript.

\section{Funding}

Medical University of Vienna.

Medical-Scientific Fund of the Mayor of Vienna (nr. 18058) - Financial project funding.

\section{Availability of data and materials}

The datasets analyzed during the current study are available from the corresponding author on reasonable request.

\section{Ethics approval and consent to participate}

Ethics approval was obtained from the Ethics Committee of Medical University of Vienna on 13 November 2017 (EK 1744/2017). The study was approved by the Austrian Federal Office for Safety in Health Care (BASG) and registered in the European Union Drug Regulating Authorities Clinical Trials Database (EudraCT Nr.: 2017-003714-68).

An investigator will obtain informed consent from all participants after checking eligibility. The study will be conducted according to the Declaration of Helsinki and Good Clinical Practice.

All changes regarding the study protocol will be communicate to the appropriate relevant parties.

\section{Consent for publication}

Not applicable

\section{Competing interests}

Not applicable

\section{Author details}

${ }^{1}$ Department of Anaesthesia, General Intensive Care Medicine and Pain Medicine, Medical University of Vienna, Spitalgasse 23, 1090 Vienna, Austria.

${ }^{2}$ Department of Surgery, Medical University of Vienna, 1090 Vienna, Austria.

${ }^{3}$ Franziskus Spital, Anaesthesia and Intensive Care, 1050 Vienna, Austria.

Received: 12 November 2019 Accepted: 23 April 2020

Published online: 12 May 2020

\section{References}

1. Devereaux PJ, et al. Association of postoperative high-sensitivity troponin levels with myocardial injury and 30-day mortality among patients undergoing noncardiac surgery. JAMA. 2017;317(16):1642-51.

2. Smilowitz NR, et al. Perioperative major adverse cardiovascular and cerebrovascular events associated with noncardiac surgery. JAMA Cardiol. 2017;2(2):181-7. https://doi.org/10.1001/jamacardio.2016.4792.

3. Pryor KO, Fahey TJI, Lien CA, Goldstein PA. Surgical site infection and the routine use of perioperative hyperoxia in a general surgical population-A randomized controlled trial. JAMA. 2004;291(1):79-87.

4. Belda JF, et al. Supplemental perioperative oxygen and the risk of surgical wound infection. JAMA. 2005;294(16):2035-42.

5. Greif $R$, et al. Supplemental perioperative oxygen to reduce the incidence of surgical-wound infection. N Engl J Med. 2000;342(3):161-7.

6. Kurz A, et al. Supplemental oxygen and surgical-site infections: an alternating intervention controlled trial. Br J Anaesth. 2018;120(1):117-26.

7. Fonnes $S$, et al. Perioperative hyperoxia-Long-term impact on cardiovascular complications after abdominal surgery, a post hoc analysis of the PROXI trial. Int J Cardiol. 2016;215:238-43. 
8. Stub D, et al. Air versus oxygen in ST-segment-elevation myocardial infarction. Circulation. 2015;131(24):2143-50.

9. Kemming Gl, et al. Hyperoxic ventilation at the critical hematocrit: effects on myocardial perfusion and function. Acta Anaesthesiol Scand. 2004;48(8):951-9.

10. Meier J, Kemming Gl, Kisch-Wedel H, Wölkhammer S, Habler OP. Hyperoxic ventilation reduces 6-hour mortality at the critical hemoglobin concentration. Anesthesiology. 2004;100(1):70-6.

11. Farquhar $\mathrm{H}$, et al. Systematic review of studies of the effect of hyperoxia on coronary blood flow. Am Heart J. 2009;158(3):371-7.

12. Shigemitsu $M$, et al. Nocturnal oxygen therapy prevents progress of congestive heart failure with central sleep apnea. Int J Cardiol. 2007;115(3): 354-60.

13. Rodseth RN. B type natriuretic peptide — A diagnostic breakthrough in perioperative cardiac risk assessment? Anaesthesia. 2009;64(2):165-78.

14. Duceppe E, et al. Canadian Cardiovascular Society Guidelines on perioperative cardiac risk assessment and management for patients who undergo noncardiac surgery. Can J Cardiol. 2017;33(1):17-32.

15. Karthikeyan $\mathrm{G}$, et al. Is a pre-operative brain natriuretic peptide or $\mathrm{N}$ terminal pro-B-type natriuretic peptide measurement an independent predictor of adverse cardiovascular outcomes within 30 days of noncardiac surgery? A systematic review and meta-analysis of observational. J Am Coll Cardiol. 2009;54(17):1599-606.

16. Rodseth RN, et al. Postoperative B-type natriuretic peptide for prediction of major cardiac events in patients undergoing noncardiac surgery: systematic review and individual patient meta-analysis. Anesthesiology. 2013;1 19(2):270-83.

17. Gan TJ, et al. Goal-directed intraoperative fluid administration reduces length of hospital stay after major surgery. Anesthesiology. 2002;97(4):820-6.

18. Feldheiser $\mathrm{A}$, et al. Balanced crystalloid compared with balanced colloid solution using a goal-directed haemodynamic algorithm. Br J Anaesth. 2012; 110(2):231-40.

19. Akça O, et al. Comparable postoperative pulmonary atelectasis in patients given $30 \%$ or $80 \%$ oxygen during and 2 hours after colon resection. Anesthesiology. 1999;91(4):991-8.

20. Meyhoff C, Wetterslev J. Effect of high perioperative oxygen fraction on surgical site infection and pulmonary complications after abdominal surgery. JAMA. 2009;302(14):1543-50.

21. Thygesen $\mathrm{K}$, et al. Universal definition of myocardial infarction (2018). Circulation. 2019;40:237-69.

22. Kirchhof $\mathrm{P}$, et al. 2016 ESC guidelines for the management of atrial fibrillation developed in collaboration with EACTS. Eur Heart J. 2016;37(38): 2893-962.

23. Devereaux PJ, Szczeklik W. Myocardial injury after non-cardiac surgery: diagnosis and management. Eur Heart J. 2019;0:1-9. https://doi.org/10.1093/ eurheartj/ehz301.

24. Ruetzler $\mathrm{K}$, et al. Supplemental intraoperative oxygen does not promote acute kidney injury or cardiovascular complications after noncardiac surgery: subanalysis of an alternating intervention trial. Anesth Analg. 2019;130(4): 933-40.

25. Kabon B, Sessler DI, Kurz A. Effect of intraoperative goal-directed balanced crystalloid versus colloid administration on major postoperative morbidity. Anesthesiology. 2019;130(5):728-44.

26. Modun $\mathrm{D}$, et al. Plasma nitrite concentration decreases after hyperoxiainduced oxidative stress in healthy humans. Clin Physiol Funct Imaging. 2012;32(5):404-8.

27. Loiseaux-Meunier MN, et al. Oxygen toxicity: simultaneous measure of pentane and malondialdehyde in humans exposed to hyperoxia. Biomed Pharmacother. 2001;55(3):163-9.

28. Liu Y, et al. Normoxic ventilation after cardiac arrest reduces oxidation of brain lipids and improves neurological outcome. Stroke. 1998;29(8):1679-86.

29. Bernardo A, et al. Effects of inflammatory cytokines on the release and cleavage of the endothelial cell-derived ultralarge von Willebrand factor multimers under flow. Blood. 2009;104(1):100-6.

30. Kiers $\mathrm{D}$, et al. Short-term hyperoxia does not exert immunologic effects during experimental murine and human endotoxemia. Sci Rep. 2015;5:17441.

31. Rousseau A, Bak Z, Janerot-Sjöberg B, Sjöberg F. Acute hyperoxaemiainduced effects on regional blood flow, oxygen consumption and central circulation in man. Acta Physiol Scand. 2005;183(3):231-40.

\section{Publisher's Note}

Springer Nature remains neutral with regard to jurisdictional claims in published maps and institutional affiliations.

Ready to submit your research? Choose BMC and benefit from:

- fast, convenient online submission

- thorough peer review by experienced researchers in your field

- rapid publication on acceptance

- support for research data, including large and complex data types

- gold Open Access which fosters wider collaboration and increased citations

- maximum visibility for your research: over $100 \mathrm{M}$ website views per year

At BMC, research is always in progress.

Learn more biomedcentral.com/submissions 